\title{
Modulating Inhibitory Control Networks in Gambling Disorder with Theta Burst Stimulation
}

\author{
Anna Marras, PhD; Gioele Gavazzi, PhD; Yogesh Rathi, PhD; Mario Mascalchi, MD, PhD; Nikolaos Makris, \\ MD, PhD; Joan Camprodon, MD, MPH, PhD; Stefano Pallanti, MD, PhD.
}
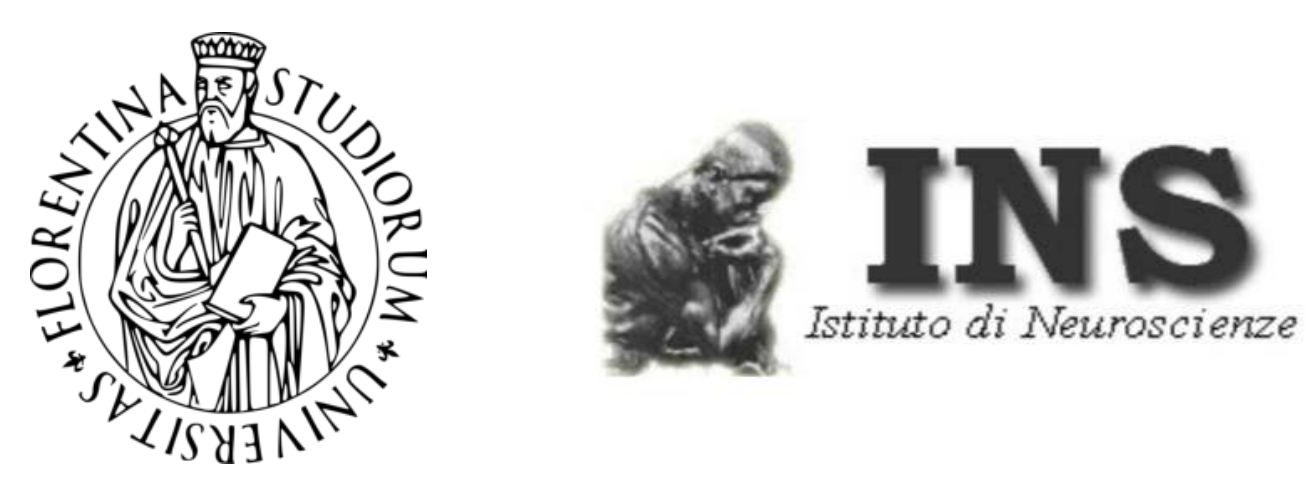

Deficits in inhibitory control from cortical cognitive systems over subcortical reward circuits is a key pathophysiological feature of addictive behavior that has not been studied satisfactorily in Gambling Disorder (GD). The presupplementary motor area (pre-SMA) is a key node of the cognitive control network responsible for response inhibition. Pre-SMA activation has been associated to response inhibition performance in FMRI studies using the SST and recent evidence also suggests that its activity represents a motivational signal for movement. Although the pathophysiology of GD is not well understood, studies have shown altered brain activity in prefrontal regions (including pre-SMA) of GD patients during response inhibition tasks in addition to functional connectivity abnormalities of SMA during rest.

In this double-blind placebo-controlled design 40 patients with GD will receive active or sham continuos Theta Burst (cTBS) to the pre-SMA for 2 weeks. We will combine TMS, multimodal structural and functional MRI and behavioral measures in order to identify circuit-level mechanisms of action and therapeutic targets (connectivity changes that explain clinical improvement) and assess the efficacy of TMS in modulating inhibitory control and symptom severity.

Stimulus intensities will be set at $80 \%$ of AMT. We will use 2 trains of 600 pulses each separated by 1 minute (a total of 1200 pulses). cTBS will be applied according to established safety guidelines. The bilateral pre-SMA will be targeted using individual MRI and a neuronavigation system as specified by Lau and colleagues (2004).
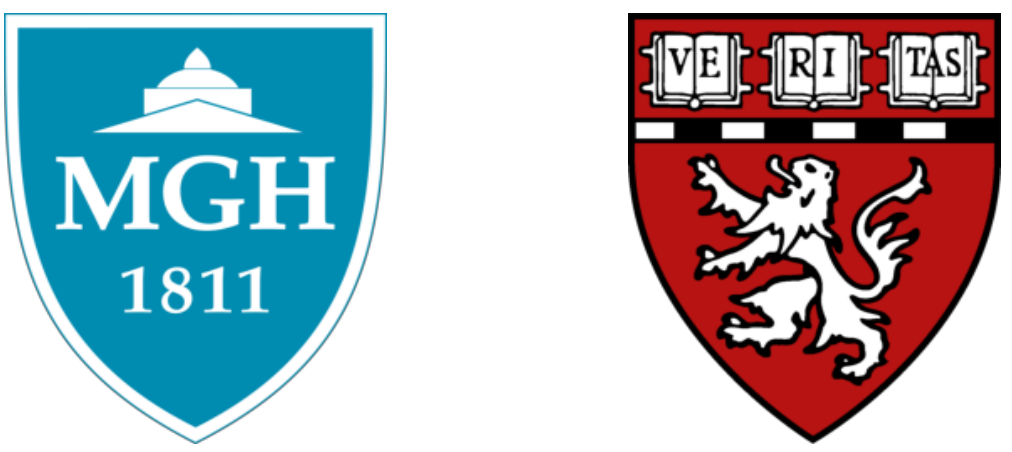

After a screening visit, the patients fulfilling the inclusion criteria will be assessed before entering the study with a comprehensive clinical interview (GD onset, duration of the illness, previous pharmacological and psychotherapeutic treatments, treatment resistance, current and past comorbidities) and a psychometric assessment.

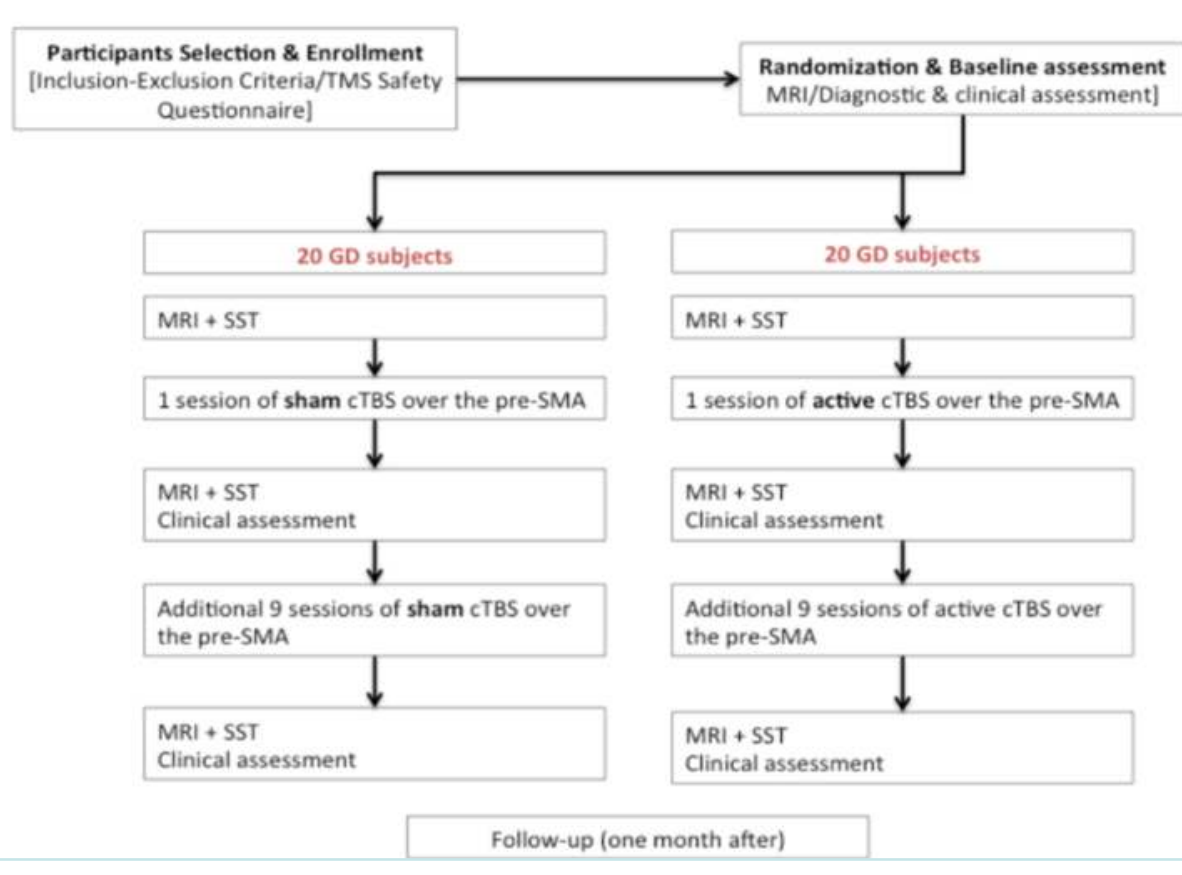

At Visit 1 patients will undergo a (pre-treatment) MRI scanning session followed by the first cTBS session. Immediately after cTBS patients will be reintroduced in the scanner for a 2nd (post-treatment) MRI. Each MRI scan will include (i) fMRI during the performance of Stop Signal Task (SST), (ii) restingstate fMRI (rs-fMRI), (iii) diffusion (dMRI) and (iv) high resolution structural T1 (in this order).

After visit 1 , which includes the first treatment, patients will receive 9 treatments additional over two weeks.

Within 2-5 days after the last reatment, patients will come for the last MRI session (also including fMRI during SST, rs- fMRI, dMRI, T1), clinical assessments and assessment of the blind. One month after treatment, there will be a last follow up session including clinical assessments.

\begin{tabular}{|c|c|}
\hline Inclusion criteria & Exclusion criteria \\
\hline $\begin{array}{l}\text { - Age: } 18-65 \text { yrs old; } \\
\text { - DSM-5 Gambling Disorder Diagnosis; } \\
\text { - Pharmacological treatment stable for } 6 \text { weeks before } \\
\text { enrollment }\end{array}$ & $\begin{array}{l}\text { - Psychiatric comorbidities except for nicotine dependence; } \\
\text { - Lifetime diagnosis of schizophrenia, psychotic disorders, } \\
\text { substance dependence or abuse, bipolar disorder I or II, } \\
\text { mental disorders due to medical condition; } \\
\text { - Suicidal risk; } \\
\text { - } \quad \text { Duration of illness < } 2 \text { yrs; } \\
\text { - } \quad \text { Hospitalization within the last } 6 \text { months before enrollment; } \\
\text { - Inability or refuse to provide written informed consent. }\end{array}$ \\
\hline
\end{tabular}

